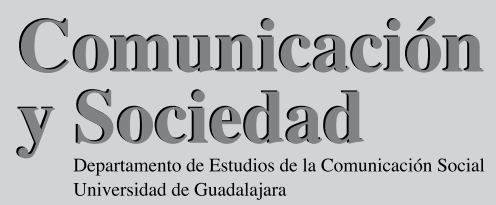

\title{
Cambios y modificaciones legislativas: repercusiones en la oferta y el contenido audiovisuales en México
}

FRANCISCO J. MARTÍNEZ G. ${ }^{1}$, EDUARDO GARCÍA R. ${ }^{2}$ Y ANDREA MENCHACA ${ }^{3}$

El trabajo hace referencia a las modificaciones legislativas que se han generado a partir de los años 90 en México, y cómo estas han influido en la oferta y el contenido audiovisual de nuestro país. El estudio, realizado con base en la técnica del análisis de contenido, enfoca su atención en el cine y en la televisión, y concluye que en esta materia el gobierno ha actuado de distinta forma en cada uno de ellos, ya que mientras por una parte se ha protegido a la industria de la televisión, por la otra se ha abandonado al cine a su suerte.

PALABRAS CLAVE: flujos de comunicación, identidades culturales, industrias culturales, reglamentación y economía política de los medios.
The work makes reference to legislative modifications generated from the 1990's in Mexico and how they have made an impact on Mexico's audiovisual media. The study, executed with a background on content analysis techniques, focuses its attention on cinema and television and concludes that, in this matter, the government of this country has not been uniform in its treatment of each since, on one hand, it has protected the television industry, and on the other it has abandoned movies to fare as they may.

KEY WORDS: communication flows, cultural identities, cultural industries, media regulation and political economy.

1 Instituto Tecnológico y de Estudios Superiores de Monterrey (ITESM).

Correos electrónicos: francisco@itesm.mx.

2 elmo_so79@hotmail.com.

3 andreamenchaca@hotmail.com. 


\section{EL INICIO DEL CAMBIO}

El primero de enero de 1994 ha quedado marcado en el calendario de México como la fecha en la cual entraron en vigor los acuerdos establecidos en el Tratado de Libre Comercio de América del Norte (TLCAN o NAFTA por sus siglas en inglés). Este mismo se venía discutiendo por autoridades canadienses, norteamericanas y mexicanas desde años atrás, resaltándose durante el proceso las ventajas que habría de acarrear a los ciudadanos de esos países la integración de un mercado común que rondaba los casi 360 millones de personas.

En tanto que los gobiernos de Brian Mulroney, George Bush padre y Carlos Salinas de Gortari resaltaban las repercusiones económicas que traería la firma del TLCAN, algunos otros ciudadanos hacían hincapié en las que podría acarrear en el sector cultural. La desconfianza partía de la mala experiencia sufrida por Canadá luego del establecimiento de un Acuerdo Bilateral celebrado con los Estados Unidos en 1987 (Silverstein, 1999, citado en Sánchez Ruiz, 1992), en el que en menos de cinco años las industrias culturales norteamericanas llegaron a controlar $93 \%$ del negocio del cine y video, $90 \%$ de la industria de discos, $92 \%$ de la edición de libros... y llegaron a obtener ganancias por más de 350 millones de dólares por las ventas de programas televisivos a Canadá.

Por ello Canadá se mostró renuente a que se incluyera en las negociaciones del TLCAN lo relacionado con el campo de la cultura, además de cualquier otra área económica, ya que suponían que la industria cultural habría de tener consecuencias importantes en la identidad cultural.

Los resultados obtenidos por Canadá incidieron, en buena medida, para impedir que en la firma del TLCAN estuvieran consideradas las llamadas industrias culturales. (ibidem:178).

La incorporación de estas en tal tratado habría acarreado tarde o temprano, para los países participantes, la obligación de eliminar todo tipo de barreras arancelarias que estuvieran ligadas a su comercialización, situación que sin duda habría causado importantes bajas a las instituciones mexicanas. Sin embargo, aun a pesar de esa mala experiencia que había afrontado Canadá, durante las negociaciones, las autoridades 
mexicanas estaban más que interesados por incorporar a las industrias culturales en el paquete del TLCAN. María de la Luz Casas (2006) considera que a los negociadores mexicanos no les importaba la cuestión relacionada con la identidad nacional, ya que, para ellos, el país cuenta con una identidad muy firme.

Así, aunque finalmente las industrias culturales no fueron incluidas dentro de la firma del TLCAN, eso no impidió que en México se realizaran una serie de modificaciones a la legislación del audiovisual, principalmente en el sector de la cinematografía y, en menor medida, en la que compete a la radio y la televisión. Bajo el argumento de que era necesaria la modificación para evitar que el país quedara rezagado en la materia, se han incorporado desde entonces una serie de modificaciones en leyes y reglamentos relacionados con el audiovisual, entre los que sobresalen los siguientes:

a) Nueva Ley de Cinematografía (1992).

b) Liquidación de la Distribuidora privada/mixta Películas Nacionales (1992).

c) Privatización de la Compañía Operadora de Teatros (1993).

d) Modificaciones a la reglamentación de la televisión por cable (1993).

e) Concluyó el proceso de privatización de la televisión mexicana (1993).

f) Modificaciones a la Ley de la Industria cinematográfica (1999).

g) Modificaciones al Reglamento de la Ley Federal de Cinematografía (2001).

h) Modificación al Reglamento de la Ley de Radio y Televisión (2002).

i) Deducción al ISR a quienes inviertan en la industria cinematográfica (2004).

Los cambios en la materia estuvieron orientados, desde un principio, a ser congruentes con el modelo de política económica que se venía promoviendo por los gobiernos de la república a partir del sexenio de Miguel de la Madrid (1982-1988). Bajo la óptica de una política neoliberal aplicada a los medios de comunicación, se reduce la burocracia, se privatizan los medios del Estado, se reducen las cuotas de pantalla, etc. Todas estas medidas eran diametralmente opuestas a las caracterís- 
ticas que distinguían hasta entonces a la política económica mexicana, cuando su tendencia era básicamente proteccionista.

Sin embargo, al entrar en vigor el TLCAN, los mecanismos modernizadores del país giraron ochenta grados para proponer la desestabilización, la desconcentración, la descentralización y por supuesto la liberación económica en medio de una situación particularmente incierta políticamente" (ibidem: 39).

A poco más de 15 años de haberse iniciado los procesos de modificación en la legislación del audiovisual en México, y ante la implementación de nuevas reglas, conviene cuestionarse sobre los resultados que estas disposiciones han traído al sector. La respuesta a la interrogante planteada pudiera tener diferentes matices dependiendo del sitio o de la óptica bajo la que se quiera analizar la situación. Sin embargo, para los académicos que ya desde hace algún tiempo remarcan el papel y la importancia que representa el audiovisual para el país, las modificaciones generadas en leyes y reglamentos del sector audiovisual han venido a "quitar paulatinamente la injerencia directa del Estado en materia de comunicación" (Crovi, 2000), a la vez que han llegado a fortalecer el modelo de comunicación-mercado, donde el sistema de medios comercial-privados se ha expandido notablemente hasta convertirse en el patrón dominante (Esteinou, 2003).

Delia Crovi (2000) sostiene, además, que los cambios que se han venido realizado a la legislación del audiovisual en México han permitido subordinar la cultura a las reglas impuestas por el mercado. Por su parte Esteinou (2003), considera que las modificaciones invitan a suponer que las políticas culturales públicas y colectivas se construyen cada vez más desde las necesidades privadas de la reproducción del capital y no desde las necesidades de avance de la población.

Desde la perspectiva de los autores señalados, los cambios que ha sufrido la legislación del audiovisual en México, desde la década de 1990, más allá de estar orientadas a la protección de la industria nacional o de crear las condiciones necesarias para su desarrollo, parecieran estar sometidas a las condiciones que han sido impuestas por parte del mercado neoliberal, situación que de ser cierta estaría lesionando gravemente el desarrollo cultural y económico del país. 
Los mexicanos lo mínimo que podríamos esperar de las modificaciones registradas en la legislación audiovisual, y más propiamente en la del cine y la televisión, es que las mismas vinieran realmente a beneficiar al sector, más no a contrarrestar su posición. Deberíamos esperar que a partir de las modificaciones se contara con una mayor participación y consolidación de la industria nacional del cine y de la televisión, a la vez que una mayor diversidad en sus contenidos y en el origen de estos, ya que no debemos olvidar que tanto la televisión como el cine son dos de las alternativas a las que más frecuentemente recurren los habitantes de este país para aprovechar su tiempo libre.

Por lo anterior, en el presente trabajo nos hemos propuesto identificar si las reformas ocurridas en sus leyes y reglamentos han impactado en la participación que mantenía en los años 90 y la que mantiene actualmente el sector audiovisual de México. El análisis de la información en el cual se habrá de basar el presente trabajo, tiene como punto de partida el año de 1994, fecha en la cual entró en vigor el TLCAN, dado que este fue finalmente el motor que vino a impulsar muchas de las reformas legislativas ocurridas en el campo audiovisual del país. Para responder al cuestionamiento anterior se han establecido una serie de preguntas:

a) ¿En que proporción ha participado el cine mexicano de la oferta cinematográfica que prevaleció y prevalece durante ambos periodos?

b) ¿Qué tan equilibrada era y es el origen de la oferta de películas que proyectaban en las salas de cine?

c) ¿Cuál es el grado de participación que tenía y tiene la oferta de televisión nacional en las cadenas nacionales?

d) ¿Qué tan equilibrada es la oferta nacional y extranjera en las cadenas nacionales de televisión abierta?

e) ¿Cuál es la proporción de contenido nacional y extranjero que se exhibe en el horario estelar de la televisión?

\section{UN RECORRIDO POR EL CINE MEXICANO}

Las conclusiones a que han llegado académicos como Sánchez Ruiz (2000a, 2003, 2004), Ugalde (2004) y Pedro Matute (2004) en torno a la participación que ha tenido el cine mexicano, coinciden al destacar la disminución que la industria ha tenido en su propio mercado 
durante los últimos años. Los argumentos a que hacen referencia giran, sobre todo, en la cada vez más limitada producción de obras, situación que no sólo lesiona la propuesta cultural a la que hacen referencia Delia Crovi y Javier Esteinou, sino que también en lo económico el país pierde una buena cantidad de recursos por la falta de participación.

La situación difícil por la que atraviesa el cine mexicano se acentúa aún más si se considera que durante algún tiempo fue una de las industrias más activas en la economía nacional. Emilio García Riera (1998) refiere que durante la década de 1940, el éxito que llegó a tener el cine mexicano alcanzó tal notoriedad, que tan sólo era superada por la industria del ensamblado de automóviles, el acero, las cervezas y los acabados de algodón.

Para Matute (2004), la participación que logró conseguir el cine nacional en la década citada por García Riera, viene a ilustrar perfectamente cómo es que esta industria podría contribuir de nueva cuenta al desarrollo económico del país. Matute refiere que el cine mexicano llegó a participar del bienestar económico de cientos de familias al crear un gran número de empleos directos e indirectos, por ejemplo, a pequeñas y medianas empresas que giraban a su alrededor.

El mal momento por el que atraviesa el cine mexicano, y al cual se hace referencia, se vislumbraba desde la década de 1980, cuando poco más de la mitad de los estrenos que se proyectaban anualmente en las salas de cine nacionales provenía de la industria norteamericana. Para principio de los años 90, la proporción de películas estadounidenses se incrementó $10 \%$, llegando a rebasar de esa manera la proporción de $60 \%$ (idem). El aumento de la oferta de películas extranjeras en el país, principalmente aquella que provenía de los Estados Unidos era de una magnitud tal, que incluso ya desde entonces el número de estas superaba la cuota que imponía la legislación cinematográfica vigente hasta entonces, la cual establecía que 50\% del tiempo en pantalla debería ser ocupado por películas nacionales.

Entre enero y septiembre de 1996, el 65\% de las cintas que se estrenaron en el área metropolitana de la ciudad de México eran estadounidenses, y por primera vez en muchos años, las producciones mexicanas no ocuparon el segundo 
lugar, sino las italianas, con el $12 \%$, las cuales rebasaron a las mexicanas quienes ocuparon el tercer sitio con un $7 \%$ del total (idem).

Al igual que los autores mencionados, Víctor Ugalde (2004) también ha considerado que el mal momento por el que atraviesa la industria cinematográfica mexicana venía ocurriendo desde dos años antes de la firma del Tratado de Libre Comercio. De acuerdo con Ugalde, desde entonces el cine mexicano mantenía un déficit comercial en relación con el de los Estados Unidos, situación que se acentúa con la reducción en la producción cinematográfica ocurrida en 1992, cuando se dejaron de exportar las 60 películas anuales que se exhibían en los estados de la Unión Americana con fuerte presencia hispanomexicana, y cuando además se tuvo que importar más películas producidas en el mercado estadounidense, a la vez que se tuvo que incrementar el número de copias para su exhibición (idem).

Sobre la participación del mercado cinematográfico nacional, Enrique Sánchez Ruiz (2000a) refiere que ya desde antes de la firma del TLC la industria extranjera, principalmente la norteamericana, superaba a la nacional no sólo en el número de cintas que se exhibían, sino también en lo económico, dado que las ganancias que arrojaba la proyección de las obras de otros sitios les otorgaban un mayor beneficio que a las mexicanas.

En 1991, de los estrenos en el área metropolitana del Distrito Federal, la mitad fueron películas estadounidenses por una cuarta parte de las mexicanas... Así las recaudaciones para 1990 en las salas del área metropolitana de la ciudad de México, según la Cámara Nacional de la Industria Cinematográfica (CANACINE) fueron en $21.5 \%$ para el cine nacional y 78.5 para películas extranjeras" (Sánchez Ruiz, 1992: 188).

La situación que atravesaba el cine mexicano presagiaba entonces una inminente catástrofe para la industria nacional, la cual, de acuerdo con Sánchez Ruiz (2000a), no era más que la antesala de su virtual desaparición, ya que las mismas reglas del mercado nos llevarían a convertirnos en meros receptores y consumidores fílmicos. Desde la década de 1980 el cine mexicano mostraba una evidente caída, mientras que la industria norteamericana cada vez participaba con mayor firmeza en México, lle- 
gando a obtener ganancias por demás considerables. Para la mitad de los años 90, poco después de la firma del TLC

los ingresos domésticos de taquilla del cine estadounidense ascendieron a 7,661 millones de dólares, a partir de 1,420 millones de entradas. Para el 2001 las taquillas estadounidenses recaudaron 8,412.5 millones, 9.8 por ciento más que el año anterior, gracias a las 1,487.3 entradas (Sánchez Ruiz, 2003: 10).

La difícil situación que se aprecia en torno a la participación que tiene el cine nacional no puede pasar desapercibida, sobre todo, porque es una verdadera lástima que contando con una serie de ventajas competitivas como estudios fílmicos, tecnología y creación cinematográfica, los cuales podrían hacer de México un polo de desarrollo cinematográfico en el mundo de habla hispana, no se haya conseguido un mejor avance en la materia. Lo que es más lamentable aún es que la capacidad instalada para realizar cine se encuentra subutilizada en $30 \%$ y que $80.27 \%$ del mercado mexicano se halla supeditado a la oferta que le programan las distribuidoras trasnacionales agrupadas a la Motion Pictures Association (Piedras, 2005).

En materia de cine, los mexicanos hemos salido perdiendo durante los últimos años, ya que hemos visto reducir el número de opciones para seleccionar películas, pues a pesar de contar cada vez con una mayor cantidad de salas de exhibición, los mexicanos

no pueden elegir libremente entre las más de cuatro mil películas de largometraje que se producen anualmente en el mundo y se tiene que resignar a escoger sólo entre las 260 cintas que le ofrecen al año, de las cuales el 63\% son de origen estadounidense (Ugalde, 2004).

Dos de las situaciones que a partir de los años 90 pudieran haber impactado más directamente en contra del desarrollo del cine mexicano son el cambio en la legislación, primero en lo concerniente a la reducción de las cuotas en pantalla y en segundo lugar la venta de COTSA, ocurrida poco tiempo después. En lo que se refiere a la reducción de las cuotas, cabe destacar que en 1992 los legisladores nacionales acordaron el esta- 
blecimiento de una disminución gradual para las obras nacionales en las cuotas de pantalla. En ese sentido, se acordó que para el año de 1993 la proporción de cintas mexicanas disminuyera de $50 \%$ a $30 \%$, y que, a la vez, esta reducción se mantuviera de manera gradual año tras año hasta que la misma quedara ubicada en $10 \%$.

Por su parte, en lo que refiere a la venta de Operadora de Teatros, S.A., en julio de 1993 el gobierno mexicano procedió a rematar un paquete de medios entre los cuales se incluyó dicha institución. De esa forma se llegó a perder la mayoría de las salas en donde se exhibía el cine mexicano, a la vez que se fortalecieron las compañías distribuidoras norteamericanas (Estrada, 2003). Con la reducción de las cuotas y la desaparición de las de pantalla, quedó abierta la posibilidad para la introducción, en 1994, de los primeros complejos cinematográficos en la ciudad de Monterrey y Aguascalientes.

Las medidas que se han implementado en la materia, además de afectar la economía del cine mexicano y del país en términos generales, también viene a repercutir - como ya se ha mencionado- en lo que se refiere al aspecto cultural, ya que la legislación propuesta, si bien contempla una reducción en la cuota de películas de origen nacional, nunca se estableció en la misma implementar una cuota que también viniera a regular el origen de las producciones, para así conseguir que los mexicanos tuviera la oportunidad de acceder a una amplia variedad de material cinematográfico producido en todos los rincones del planeta y no estar sujetos solamente a uno, como ha sido denunciado por los académicos y cineastas del país.

\section{¿Y QUÉ HA SIDO DE LA TELEVISIÓN?}

En lo que hace a la televisión, el Estado mexicano ha venido actuando de una manera por mucho más cautelosa que como ha sucedido en el cine, ya que las reformas legislativas que se han implementado en torno a tal medio han sido más protectoras. Aun así, las modificaciones realizadas a la legislación de la televisión también presentan características neoliberales; especialmente, sobresale este detalle por la privatización de la empresa de televisión pública IMEVISION ocurrida en julio de 1993 . 
Ni siquiera la venta de IMEVISION vino a concluir con los constantes conflictos en los cuales la televisora pública se vio envuelta prácticamente desde su nacimiento. Esta televisora era la única de la red pública que mantenía alcance nacional, fue dirigida durante más de veinte años por el Estado mexicano y para proceder a su venta el gobierno se vio en la necesidad de regularizar la situación legal de los canales 7 y 13, los cuales se encontraban sometidos al régimen de permisionarios, situación que en términos legales impedía su venta.

Debido al largo monopolio que en México había ejercido hasta entonces la empresa TELEVISA, a la naciente TV Azteca se le otorgaban pocas esperanzas de desarrollo. No obstante, con base en una política agresiva, buscó asociarse - sin éxito- con la NBC; hizo alianza con Telemundo en materia de producciones y artistas, y cambió su programación, dándole a esta un tinte amarillista y violento (Crovi, 2000). En su etapa inicial TVAzteca produjo telenovelas con argumentos que en su momento llegaron a ser muy controvertidos, aunque con una gran aceptación, y el 8 de agosto de 1997 concretó un audaz movimiento económico al ofertar $29 \%$ de su capital social en la bolsa de valores, proporción que en términos reales equivalía a 640 millones de dólares, cantidad similar al pago que hicieron sus dueños por la misma empresa apenas cuatro años atrás.

Alfredo Enríquez sostenía, en 1995, que la venta de IMEVISION, aunado a la firma del Tratado de Libre Comercio y a la implementación de políticas económicas neoliberales por parte del gobierno federal, no traerían más que presagios negativos para la televisión mexicana. El autor consideraba que la apertura comercial del Tratado de Libre Comercio de América del Norte habría de promover la constante inclusión de programación de origen estadounidense en la agenda de los canales de TVAzteca.

Sin embargo, la situación fue distinta a lo expresado por Enríquez (2005), ya que durante los años 90 el origen de los programas de televisión que se transmitían por la televisión abierta del país mostraban una tendencia sostenida, la cual apuntaba directamente hacia su mexicanización. Sin embargo, esta pauta se revirtió abruptamente en 1995, llegando a recuperarse ligeramente para el año de 1997 (Sánchez Ruiz, 2000b). La razón del porqué se presentó un incremento en la transmisión de contenidos extranjeros durante el periodo señalado, obede- 
ció, de acuerdo con Sánchez Ruiz (idem), a que para entonces se había desatado la competencia entre TVAzteca y TELEVISA, y que durante ese año la empresa recién conformada importó, además de programas norteamericanos, una buena cantidad de telenovelas latinoamericanas (idem).

Otro de los sucesos relacionados con la participación de la televisión mexicana durante el periodo de estudio, está ligada a la reforma efectuada al Reglamento de la Ley de Radio y Televisión ocurrida en 2002. En lo que concierne a la legislación relacionada con la televisión, de hecho se esperaban modificaciones importantes directamente en la Ley, la cual, entre otras cosas, había sido acusada de estar muy desfasada en lo tecnológico, dado que este databa de 1960. Sobre el particular Esteinou remarcaba (2005) que a 45 años de la promulgación la Ley Federal de Radio y Televisión en el país, ya se habían presentado profundos cambios económicos, políticos, sociales, demográficos, tecnológicos y culturales, los cuales modificaban sustancialmente la estructura de la sociedad mexicana, por lo que proponía la realización de una profunda reforma legal en ese terreno para actualizarlo a la realidad contemporánea, de lo contrario -afirmaba Esteinou- se seguiría contando con una industria de la comunicación que actúa en grandes vacíos jurídicos.

Sin embargo, aun a pesar del rezago en la legislación de televisión, la participación del presidente Vicente Fox se limitó a la conformación de una Mesa del Diálogo para la Reforma a la Ley de Radio y Televisión, la cual reunió durante varios meses a grupos relacionados con la academia, la política, la sociedad civil, los empresarios y los trabajadores de la radio y la televisión. No obstante el 10 de octubre de 2002, en un movimiento que tomó por sorpresa a la mayoría de los integrantes de la llamada Mesa del Diálogo para la Reforma de la Ley de Radio y Televisión, el presidente Fox firmó con los empresarios del sector una modificación al reglamento de la mencionada ley, mismo que fue duramente criticado, sobre todo porque en el decreto del 10 de octubre, además de quedar fuera la mayor parte de las propuestas presentadas por los integrantes de la Mesa de Diálogo, se vendría a reafirmar una vez más los beneficios que obtiene el sector empresarial gracias al mercado audiovisual. Entre otros, se destaca la eliminación 
del decreto de $12.5 \%$ de tiempos fiscales que existía hasta entonces, de tal manera que en adelante los empresarios de la radio y la televisión estarían obligados solamente a proporcionar dieciocho minutos diarios de transmisión, en el caso de estaciones de televisión, y 35 minutos diarios en las de radio.

De hecho, una buena parte de las críticas que se dieron en aquel momento estuvieron orientadas a desacreditar la acción del presidente Vicente Fox, al cual criticaron no sólo por haber actuado en favor de empresarios del medio al momento de extender el nuevo Reglamento, sino también por las acciones y situaciones que habrían de continuar al mencionado acontecimiento:

También se ufana el Presidente de incluir a "la sociedad civil" en el Consejo Nacional de Radio y Televisión. Pero tomará asiento en él un solo representante de la dicha sociedad, con voz pero sin voto, en un órgano integrado por los representantes de cuatro secretarías de Estado, tres de los concesionarios y dos de los trabajadores de la radio y la televisión. En este punto (artículo 47 del reglamento), el populismo de Echeverría (autor del reglamento de 1973) fue sustituido por el empresarismo de Fox: en ambos casos yendo más allá de la ley, convertidos por lo tanto en legisladores espurios, ambos presidentes añadieron un miembro más al Consejo. La ley estipula que sean dos representantes de los industriales y dos de los trabajadores. Pero Echeverría dispuso que hubiera "un representante más de los trabajadores", con lo que se rompió la paridad querida por la ley. Ahora se ha roto de nuevo, pero en sentido contrario, pues Fox ordena que en el Consejo haya "uno más de la industria de radio y televisión". Quizá se quiso que hubiera tres y tres, pero habrá sólo dos de los trabajadores ya que la adición de 1973 quedó abrogada como el reglamento todo que la contenía (Granados Chapa, 2002: 17).

En ese sentido, lo que ha ocurrido en el sector de la televisión contrasta con la política que los gobiernos de la república han puesto en marcha para con el cine mexicano durante todo este tiempo, ya que mientras que con este último la participación ha estado orientada hacia la implementación de una postura neoliberal, en torno a la televisión se ha implementado una política legislativa más proteccionista en favor de los empresarios del ramo a quienes todo esto les ha resultado sumamente benéfico. 
La situación que se ha descrito en torno a los privilegios que rodean a los empresarios del ramo de la televisión puede explicar la razón por la cual los contenidos nacionales han venido superando a los que proceden del extranjero, según lo indican los estudios realizados por Delia Crovi y Enrique Sánchez Ruiz.

A mediados de la década de 1990 Delia Crovi encontró que en términos generales 58\% del tiempo que ocupaban los contenidos de televisión habían sido producidos en México, y el resto procedía del extranjero, principalmente de los Estados Unidos (1995:186). Por su parte Sánchez Ruiz (1995) en un trabajo similar realizado en la ciudad de Guadalajara, encontró que la oferta nacional ocupaba 53\% de tiempo de transmisión y que 33\% de la misma provenía de los Estados Unidos. Sin embargo, este autor resalta (ibidem: 206) que en la programación del horario estelar (AAA) la participación nacional se reduce $(46 \%)$, mientras que se incrementa en cinco puntos porcentuales la participación de contenidos originados en los estados Unidos.

En otro trabajo similar, Sánchez Ruiz (2000b), destaca que durante el horario estelar es cuando se transmite en la televisión mexicana la mayor parte de la programación norteamericana, mientras los contenidos nacionales decrecen. En especial, este detalle se aprecia en las películas, las cuales, a decir del mismo autor, habían llegado a ocupar el sitio que anteriormente era llenado por producciones mexicanas.

\section{MÉTODO DE TRABAJO}

El análisis de la oferta programática que se realizó tanto para el cine como para la televisión fue realizado con base en la técnica del análisis de contenido cuantitativo. Sin embargo, existen notables diferencias metodológicas en el empleo que se hace de la misma técnica, sobre todo en lo relativo al muestreo y al tamaño de la muestra.

Para la realización del análisis de la oferta cinematográfica se conformó una muestra aleatoria que comprendió un total de diez lunes, los cuales a su vez fueron obtenidos del universo que comprende las 52 semanas del año. La decisión de seleccionar la muestra de películas exclusivamente de la cartelera que se publicaba los días lunes, se debe básicamente a que en México, en la mayoría de los casos, las carteleras 
cinematográficas son cambiadas los días viernes y se mantienen hasta el día miércoles o jueves de cada semana.

Las películas que fueron incluidas en el estudio parten exclusivamente de la oferta programática que se presentó en las salas de cine de la ciudad de México, y que a la vez aparecieron publicadas en los diarios La Jornada, para aquellas que se exhibieron durante 1994, y el diario Reforma para la muestra que comprende el año 2004. Finalmente, para la conformación de la ficha técnica, en donde se incluye el origen de las mismas, se recurrió a la página electrónica de Internet Movie Data Base (IMDB).

Para el estudio relacionado con los contenidos de la televisión, se recurrió a la conformación aleatoria de dos semanas completas para cada uno de los años incluidos en el estudio y en las mismas se abarca el universo de los 365 días del año. Los programas incluidos en el trabajo representan exclusivamente a los que se transmitieron en los canales nacionales de la televisión abierta: Canal 2, 5 (Televisa) y Canal 7 y 13 (TVAzteca). El criterio en el cual se sustentó la selección de estos canales de televisión tiene que ver, en primer lugar, con el rating que cada uno de ellos presenta, y, en segundo, con base en la penetración y alcance que tienen en el territorio nacional.

El análisis comprendió únicamente la oferta programática que se difundió a partir de las 6 de la mañana y hasta las 24 horas. Sin embargo, se tomó el criterio de incluir el tiempo total que duraba la transmisión de un determinado programa, esto es, aun cuando el mismo haya sobrepasado el horario límite al cual se ha hecho referencia. Por ejemplo, si un determinado programa iniciaba a las 23:30 hrs y concluía a las 0:30, en todos los casos esos 30 minutos que rebasaban el tiempo límite, que para el caso había sido fijado a las 24 horas, también se contabilizaba ${ }^{4}$.

4 Este criterio finalmente se habrá de reflejar en el tiempo total de minutos de transmisión que aparecen en los resultados, ya que en muchas ocasiones los programas que se transmitían a última hora del día solían rebasar el tiempo límite. Este mismo criterio también fue utilizado en aquellos programas que se proyectaban durante el horario estelar. 


\section{LA OFERTA CINEMATOGRÁFICA}

Durante el 2004 la cantidad de películas que se exhibieron en las salas de cine de la ciudad de México superó en casi $100 \%$ a la oferta que existía en 1994. En buena medida, esta situación obedece al incremento en el número de salas cinematográficas merced a la consolidación del modelo de complejos cinematográficos que han surgido en la ciudad capital del país. La gran cantidad establecimientos de este tipo ha permitido que se incremente en casi $750 \%$ el número de funciones de cine que se ofrecen en la ciudad de México, ya que mientras que en 1994 se proyectaban en promedio 483 funciones diariamente, diez años después la cantidad se disparó hasta las 3611 funciones.

Sin embargo, aunque en la actualidad la cantidad de exhibiciones que se generan diariamente supera por un amplio margen a la que tenía lugar en 1994, el número de títulos de películas que se presentaban entre uno y otro periodo, no supera ni siquiera al $100 \%$, ya que mientras durante 1994 se presentaron 126 películas diferentes, para el 2004 la cantidad fue de 235. La situación anterior tiene su explicación nuevamente en el incremento que se ha generado en las salas cinematográficas de la ciudad de México.

En lo que se refiere a la presencia de películas mexicanas en las mismas salas, es posible reparar en la existencia de una notable diferencia en lo que concierne al número de títulos con los cuales se participó durante 1994 y la que se presenta diez años después. Así, mientras que en 1994 la presencia del cine mexicano estuvo representada por 28 cintas, para el 2004, la cantidad disminuyó hasta ubicarse en ocho. La situación descrita significa que en diez años la presencia de películas mexicanas ha disminuido su participación en poco más de $350 \%$ en las pantallas de la ciudad capital del país. Pero lo que es más, si se enfoca la atención en la proporción de cintas nacionales que se exhibieron entre uno y otro año, la disminución se hace aún más evidente, puesto que en 1994, 22\% de las películas que aparecieron en la cartelera habían sido originadas en el México, para el 2004 la misma proporción apenas si alcanza $3 \%$.

La disminución que ha tenido en su participación el cine nacional en las carteleras de la ciudad de México, ha repercutido de una forma tal 


\section{TABLA 1}

ORIGEN DE LAS PELÍCULAS QUE SE EXHIBIERON

DURANTE EL PERIODO ANALIZADO

País productor

Periodo analizado

\begin{tabular}{lcc} 
& 1994 & 2004 \\
\hline Estados Unidos & $58 \%$ & $49 \%$ \\
Francia & & $7 \%$ \\
México & $22 \%$ & $3 \%$ \\
España & $2 \%$ & $3 \%$ \\
Canadá & & $2 \%$ \\
Reino Unido & & $2 \%$ \\
Dinamarca & & $1 \%$ \\
Alemania & $2 \%$ & $1 \%$ \\
Italia & $4 \%$ & \\
Otro(s) & $12 \%$ & $32 \%$ \\
Total & $100 \%$ & $100 \%$ \\
& $(\mathrm{n}=126)$ & $(\mathrm{n}=235)$
\end{tabular}

* La proporción de películas que se contemplan en este apartado incluye a las coproducciones, las cuales se multiplicaron para el segundo periodo analizado.

que a diferencia de lo que ocurría en 1994, cuando ocupaba el segundo sitio, ha pasado a ocupar, para el 2004, un tercer lugar, siendo rebasado por la industria cinematográfica francesa, la cual aportó casi 5 puntos porcentuales más que su parte mexicana.

Los resultados señalados, además de mostrar una evidente disminución en la cantidad de cintas nacionales, también parecen mostrar que cada vez, en las salas de cine de la capital del país, la proporción de cintas originadas en los Estados Unidos es menor, ya que de 58\% que ocupaban en 1994, decrecieron hasta participar con $49 \%$, esto es, nueve puntos porcentuales abajo. Sin embargo, a pesar de que la situación se ha presentado así, no han afectado en lo mínimo a la industria norteamericana, primero porque a diferencia de lo que ocurría en 1994, en la actualidad esta industria es la que mayor participación 
tiene con base en coproducciones con industrias de otros países. Luego, bajo esa óptica, si se considera no sólo las producciones propias, sino que a estas se les añade la participación a través de las coproducciones, el incremento de la industria norteamericana del cine sería de alrededor de cinco puntos porcentuales. Es decir, bajo esta óptica, $54 \%$ de las películas que se exhibieron durante 2004 tenían relación también con el mercado cinematográfico estadounidense.

Pero, además, la diferencia que existe en la proporción de películas norteamericanas que se proyectaron, en comparación con aquellas que han sido producidas en otros lugares del orbe, difiere sustancialmente si se repara también en el promedio de tiempo que cada una de las cintas se mantiene en cartelera durante los dos periodos analizados, así como en el número de salas en las que estas se proyectan. Bajo esa mirada, es evidente que las películas norteamericanas participan de una mayor cantidad de tiempo en la cartelera, a la vez que sus obras son exhibidas en una mayor cantidad de salas, incluyendo las películas mexicanas, situación que ha sido también ya señalada entre otros autores por Martínez y Lozano (2000).

El desequilibrio que existe entre la exhibición que tienen las obras que han sido producidas en los Estados Unidos y las que provienen del resto de las naciones del mundo en las carteleras cinematográficas de la ciudad de México, se hace presente prácticamente bajo todos los órdenes que rodean al análisis. Y es que en el caso del cine norteamericano, no sólo son más las cintas que tienen relación con el cine de aquella nación, sino que estas mismas cintas son, a la vez, las que se exhiben en una mayor cantidad de salas y las que se mantienen presentes en la cartelera durante mayores periodos de tiempo (ibidem: 61-62).

En ese sentido, si se repara en la participación que tiene en la cartelera cinematográfica cada uno de los países, se hará más notable tanto el decrecimiento que ha tenido la industria mexicana, así como el incremento que ha tenido el mercado norteamericano. En el primero de los casos se presenta una disminución de $5 \%$ en la cantidad de funciones contra un incremento de $3 \%$ en la programación de las norteamericanas (tabla 2). 
En otras palabras, la industria mexicana del cine participó con un menor número de títulos en la muestra obtenida durante el 2004, en comparación con la participación que tuvo en 1994, pero, a la vez, en promedio sus cintas también se programaron en las salas en una proporción menor, de tal manera que anteriormente existía una mayor probabilidad de encontrar en cartelera una película mexicana que hoy en día. Todo ello pone en evidencia la poca atención que han prestado al sector cinematográfico tanto el Ejecutivo como los legisladores. Los resultados muestran que se ha hecho a un lado la importancia y relevancia del mismo, tanto en lo económico como en lo cultural, esto es, a pesar de que en la Ley de Cinematografía de este país se advierte, desde el inicio, un apartado donde se destaca la leyenda que reconoce al cine como un sector de vital importancia para México.

Diariamente, en las salas de cine de la capital del país, las películas norteamericanas mantienen una participación en cartelera equivalente a ocho de cada diez películas. Es decir, sólo dos películas de las que se exhiben en esta ciudad no están ligadas a la industria de los Estados

\section{TABLA 2}

PROMEDIO DE EXHIBICIONES CON LAS CUALES PARTICIPA

EN EL MERCADO NACIONAL CADA UNO DE LOS PAÍSES

País de origen

$\%$ funciones

\begin{tabular}{lcc} 
& \multicolumn{2}{c}{ Periodo analizado* } \\
\hline & 1994 & 2004 \\
México & $12 \%$ & $7 \%$ \\
Estados Unidos & $77 \%$ & $80 \%$ \\
Francia & $2 \%$ & $5 \%$ \\
España & $2 \%$ & $7 \%$ \\
Canadá & $0 \%$ & $1 \%$ \\
Reino Unido & $2 \%$ & $6 \%$ \\
& $(\mathrm{n}=4829)$ & $(\mathrm{n}=36119)$ \\
& & \\
La proporción de películas que se contemplan en este apartado incluye a las \\
$\quad$ coproducciones, las cuales se multiplicaron para el segundo periodo anali- \\
zado.
\end{tabular}


Unidos. Lo anterior pone en evidencia que los productos de dicho país mantienen un beneficio en la distribución, ya que estas se programan en una mayor cantidad de salas, a la vez que se ofrecen, en promedio, en una mayor cantidad de funciones.

La situación anterior viene a corroborar la postura que en torno al cine nacional han mantenido autores como Matute (2004), Ugalde (2004) y Sánchez Ruiz (2000a, 2004), los cuales han denunciado la situación que presenta el sector, dejando en claro que en México la única industria cinematográfica que presenta una sólida consistencia en su crecimiento ha sido la de los Estados Unidos, la cual presenta una destacada participación cuando de exhibir sus productos se trata, situación que contrasta con lo que ocurre con las cintas nacionales.

Lo anterior viene a demostrar no sólo el dominio que en México tiene la industria cinematográfica del vecino país, sino también permite constatar que aunque existen otros países que ofertan sus productos en el mercado mexicano, en la mayoría de los casos no tienen el mismo trato que las cintas que provienen del mercado norteamericano, o que bien están relacionadas con este mismo.

\section{LA SITUACIÓN EN LA TELEVISIÓN}

Desde 1994 y hasta el 2004, en la programación de la televisión mexicana se han venido presentando una serie de modificaciones en los contenidos, entre las que se destaca el incremento del tiempo que se dedica actualmente a temáticas que tradicionalmente recibieron poca atención. Uno de ellos tiene que ver directamente con el que se destina al macrogénero relacionado con los informativos. Este, por sí mismo, pasó de 15 a $22 \%$ en el tiempo total de la transmisión de los canales de la televisión nacional abierta. Esto significa que los programas que están relacionados con esta categoría, como lo son los noticieros, magazines, programas de entrevistas, etc., han recibido un tiempo mayor en su cobertura. También se recibió un notable incremento en el tiempo que en promedio se destinaba a los programas relacionados con telemercadeo. En este último caso, el incremento podría sin duda ser aún más notable si se incluyera en el estudio, también, el horario 
que va más allá de la medianoche5, ya que ahí es justo en donde este tipo de programas son presentados con mayor frecuencia.

Se mantiene entre uno y otro periodo la presencia sobresaliente del género de ficción (tabla 3), el cual ocupa en ambos casos un tercio del tiempo total de transmisión, y donde se destaca la presencia de películas y telenovelas, dos de los géneros con mayor participación en las pantallas de los canales de la televisión nacional, de tal manera que por sí mismos cubren -en ambos periodos- poco más de la cuarta parte del tiempo que se destina a la transmisión de programas en la televisión mexicana $y$, por el contrario, existe una disminución en el tiempo que se destina a los programas de variedades.

\begin{tabular}{lcc}
\hline \multicolumn{1}{c}{ TABLA 3 } \\
\multicolumn{1}{c}{ PROPORCIÓN DE TIEMPO QUE DESTINARON LAS TELEVISORAS } \\
& A CADA UNO DE LOS MACROGÉNEROS \\
Macrogéneros & $\%$ & $\%$ \\
& 1994 & 2004 \\
\hline Información & 15 & 22 \\
Ficción & 36 & 33 \\
Infoshow & 3 & 4 \\
Variedades & 14 & 7 \\
Infantiles & 19 & 17 \\
Deportes & 8 & 7 \\
Culturales & 2 & 1 \\
Telemercadeo & 2 & 8 \\
Programa político & 2 & $100 \%$ \\
Total & $100 \%$ & $(\mathrm{n}=1002 \mathrm{hrs})$. \\
\hline
\end{tabular}

En lo que corresponde los contenidos informativos, los cuales ocupan uno de los mayores tiempos en las pantallas nacionales, se destaca la existencia, entre 1994 y 2004, de un incremento de alre-

5 El tiempo de análisis abarca exclusivamente de las 6 am a las $12 \mathrm{pm}$, como ha sido descrito en la metodología del trabajo. 
dedor de siete puntos porcentuales. El resultado anterior obedece al tiempo que más recientemente se le destina a los denominados magazines, más que al tiempo que se destina a los telenoticieros. En ese sentido, mientras que en el periodo de 1994, los noticieros ocupaban $12 \%$ del tiempo total de la transmisión en las televisoras nacionales, para el 2004 el espacio se había reducido cinco puntos porcentuales, hasta ubicarse en 7\%. Esta situación de los noticieros de televisión es contraria a la que se presenta en torno a los magazines o programas de revista, donde el tiempo que se les destinó pasó, en ambos periodos, de 2 a 14\% en 2004 (tabla 4).

Contrario a lo que pudiera haberse pensado, a diez años de la firma del TLCAN, los contenidos que se transmiten en la televisión mexicana son, tal y como viene ocurriendo desde 1994, en su mayor parte originados en el propio país. La presencia de programas nacionales es mayor no sólo en el número de programas, sino también en el tiempo que se destina a los mismos.

Sin embargo, en el caso de ambos periodos se hace más que evidente la poca presencia de programación que ha sido originada en países diferentes a México o a los Estados Unidos, de tal manera que la participación de terceros países se ha mantenido prácticamente igual desde entonces. Y si se quisiera reparar en alguna diferencia, sin duda que esta tendría que ver con la disminución de los productos latinoamericanos en la televisión mexicana. Así, mientras que en 1994 en México era posible encontrar en la agenda programas originados en Argentina y Venezuela, apenas una década después la del primero de los países mencionados ya no está presente, y en el caso de Venezuela su presencia se ha reducido a la mitad.

El tiempo que los productos nacionales mantienen en pantalla se ha incrementado, de 1994 a 2004, en 5\%. Sin embargo, aun cuando la presencia de contenidos nacionales supera a la de cualquier otro país, estos presentan muy poca variedad. Así, durante 1994, las 2/3 partes del tiempo que se destinaba a la transmisión de programas nacionales, consistían tan sólo en noticieros (19\%), películas (17\%), musicales (13\%), telenovelas $(10 \%)$ y a la transmisión de eventos deportivos $(8 \%)$. Tal situación no parece mejorar mucho para el 2004, cuando la mayor parte de los contenidos nacionales consisten en magazines (22\%), telenove- 


\section{TABLA 4}

PROMEDIO DE TIEMPO DADO A CADA UNO DE LOS GÉNEROS

\begin{tabular}{|c|c|c|}
\hline Género & $1994(\%)$ & $2004(\%)$ \\
\hline Actividades y concursos & 4 & 2 \\
\hline Comentarios, análisis, entrevistas de deporte & 1 & 4 \\
\hline Cómicos & 1 & 1 \\
\hline Dibujos animados & 15 & 13 \\
\hline Documentales & & 1 \\
\hline Entrevistas y debate & 2 & 1 \\
\hline Humorísticos & 1 & 2 \\
\hline Juegos y concursos & 4 & 2 \\
\hline Magazines & 2 & 14 \\
\hline Musicales & 8 & 2 \\
\hline Noticiero & 12 & 7 \\
\hline Película & 15 & 16 \\
\hline Programas de ventas directas al público & 1 & 8 \\
\hline Reportajes & 1 & 1 \\
\hline Serie de ciencia ficción & & 1 \\
\hline Serie de comedia & 3 & 1 \\
\hline Serie de drama & 3 & 1 \\
\hline Series de aventura y acción & 3 & \\
\hline Series infantiles & & 1 \\
\hline Talk shows & 2 & 2 \\
\hline Telenovelas & 10 & 11 \\
\hline Televerdad & & 1 \\
\hline Transmisión de juegos o competencias & 7 & 3 \\
\hline Videos de aficionados & & 1 \\
\hline \multirow[t]{2}{*}{ Otro género } & 5 & 4 \\
\hline & $100 \%$ & $100 \%$ \\
\hline Total & $(\mathrm{n}=875$ hrs. $)$ & $(\mathrm{n}=1002$ hrs. $)$ \\
\hline
\end{tabular}

las $(15 \%)$, telemercadeo $(12 \%)$, noticieros $(11 \%)$ y programas relacionados con los comentarios deportivos $(6 \%)$.

Además del tiempo de transmisión que se le concedió durante el 2004 a los magazines, y del cual se ha hecho referencia, existe otra marcada 
diferencia en lo que concierne a la transmisión de películas. En términos generales, para 1994 la mayoría del tiempo (66\%) que se destinaba a la transmisión de películas estaba siendo llenado básicamente con cintas de procedencia nacional; es decir, durante los fines de semana las televisoras mexicanas tendían a programar principalmente cintas extranjeras $(56 \%)$, pero la presencia nacional se encontraba presente. Sin embargo, para el 2004 ni tan siquiera entre semana, ni mucho menos los fines de semana se destaca la presencia de películas mexicanas en ese medio.

Durante el horario estelar, la situación que prevalecía en 1994 dista mucho de ser similar a la que se presenta diez años después. En el primero de los periodos incluidos en el estudio, el tiempo de programación con base en contenido de origen estadounidense rebasa muy ligeramente a la nacional. Esta situación se debía a la presencia de programas relacionados con películas, series de comedia, de acción, de drama y miniseries, las cuales, en la actualidad, en buena medida han sido desplazadas por contenidos nacionales. En el último periodo incluido en el estudio, los programas nacionales ocupan dentro del horario estelar poco más de las 2/3 partes del tiempo de transmisiones (tabla 5).

\section{TABLA 5}

TIEMPO CON EL CUAL PARTICIPA CADA UNO DE LOS PAÍSES

EN EL HORARIO ESTELAR DE LA TELEVISIÓN MEXICANA

\begin{tabular}{lcc} 
País de origen & \% de tiempo & \% de tiempo \\
& 1994 & 2004 \\
\hline Estados Unidos & 47 & 31 \\
México & 44 & 67 \\
Japón & 3 & 2 \\
Brasil & 3 & \\
España & 1 & \\
Canadá & 1 & \\
Venezuela & 1 & $100 \%$ \\
Total & $100 \%$ & $\mathrm{n}=238 \mathrm{hrs}$. \\
\hline
\end{tabular}




\section{CONCLUSIONES}

Ante una situación como la anteriormente descrita, se puede concluir que aquellos que de antemano han reparado en la mala etapa por la que atraviesa el cine nacional están en lo cierto y, que incluso cuando los poderes Legislativo y Ejecutivo en México hayan implementado modificaciones a su legislación, y la hayan considerado como una industria prioritaria para el país, los resultados encontrados en el análisis vienen a demostrar que en realidad se ha retrocedido desde hace una década.

Si mediante la modificación a las reglas del juego se quiso contribuir al desarrollo y al fortalecimiento de la industria del cine mexicano, la evidencia apunta que al menos, hasta el momento, ello no se ha logrado alcanzar, y que por el contrario, la venta de COTSA y la disminución en las cuotas en pantalla ha venido a beneficiar a quienes ahora se han hecho del control de la industria del cine en México.

Con la venta de COTSA, el gobierno de la república se olvidó de implementar un plan que viniera a apoyar la distribución del material fílmico originado por las industrias del país. Así mismo, se dejó pasar la oportunidad de participar, mediante esta industria, en dos importantes aspectos que inciden directamente en la vida de México: primero, dejamos de difundir la cultura nacional y, segundo, también hemos hecho a un lado la oportunidad de participar activamente en favor de nuestra economía. Quien se encargaba de distribuir el cine nacional ya desapareció; las salas de cine se han borrado de aquellos lugares en los cuales el número de la población o los recursos económicos no son suficientes para satisfacer los intereses de quienes se han dedicado a la distribución del cine en el país. Además, las cuotas que han sido impuestas desde la misma Cámara de representantes del Poder Legislativo (las cuales de por sí son reducidas) no se cumplen, ni en lo que respecta a la proporción de películas ni a la exposición de las mismas, y aparentemente no existe quien repare en ello.

Contrario a lo que deberíamos de esperar, para el 2004 la participación que tiene la industria cinematográfica de México presenta una disminución en la participación del mercado, esto en comparación con la que mantenía diez años atrás. Esto significa que en lugar de mejorar la situación del sector, su participación es cada vez más limitada. En este 
caso, las autoridades gubernamentales deberían preocuparse por conseguir que la sociedad mexicana tenga una mejor oferta, porque, independientemente del modelo económico que se ha venido imponiendo, no puede limitarse a una dieta cultural tan pobre al público del cine.

Actualmente, la oferta cinematográfica que existe denota mucha pobreza, ya que al público de este país no le queda más opción que la de sujetarse a las imposiciones del mercado, las cuales consisten básicamente en cintas norteamericanas. Carecemos de variedad de origen y nos privamos, así, de la oportunidad de acceder a otro tipo de contenidos. Las modificaciones que se han efectuado a la legislación del cine, en México, han traído consigo una imposición terrible, la cual impide a sus ciudadanos tener un mayor número de opciones donde disfrutar del medio.

Aunque las industrias culturales no fueron incluidas en el TLCAN, las autoridades mexicanas procedieron a reformar aspectos legislativos relacionados con las mismas y, bajo las nuevas condiciones, los resultados dejan entrever que en las salas de cine de la ciudad capital del país se exhiben ocho películas norteamericanas por dos del resto del mundo. Lamentablemente, no existe ninguna duda de que las aseveraciones que hicieran en su momento Esteinou, Crovi, Sánchez Ruiz, Ugalde y Matute sobre el cine mexicano, vienen a coincidir plenamente con los resultados obtenidos en este análisis.

La situación es distinta en la televisión, debido a que este medio se encuentra, como de hecho ha estado desde su inicio, demasiado protegido. Como evidencia de ello cabe destacar que las modificaciones que se han realizado en torno a su legislación, desde siempre han quedado pendientes, y aquellas que se han concretado han estado orientadas hacia la protección de los grupos que se han visto favorecidos con una concesión.

Los cambios que se han generado en su reglamento ya tienen sus consecuencias en el tipo de contenidos que se presentan al público. El más notable tiene que ver con el tiempo destinado al telemercadeo, el cual ha pasado del 1\%, en 1994, a 8\% para el 2004.

En la programación de la televisión nacional abierta los contendidos locales superan en cantidad y tiempo de exposición a los que proceden del exterior, pero aun así, bien vale la pena discutir sobre este particular. Primero porque una de las características que distinguen a los productos 
nacionales suele ser la poca inversión que se destina a su realización, como sucede con el género de los magazines, los programas de entrevistas y la compra de películas norteamericanas.

En el mejor de los casos se invierte en la producción de telenovelas, mismas que se han apropiado del horario nocturno, evidenciando la pobre variedad que existe en la programación de la televisión nacional abierta. También se han venido generando una serie de programas cuyo mayor mérito tiene que ver con que estos son copias de fórmulas probadas en el extranjero. Es decir, en la televisión mexicana sí predomina la programación realizada en sus televisoras, pero las favorables condiciones bajo las cuales se desenvuelven los empresarios del medio, les permite recurrir a la implementación de fórmulas baratas sin tener que temer por la competencia.

Por lo anterior, valdría la pena discutir muy seriamente si la televisión mexicana es buena tan sólo porque la mayor parte de sus contenidos son mexicanos, sobre todo cuando reparamos en la falta de variedad que los distinguen.

Por último, valdría la pena, también, enfocar la atención en el origen de las películas que se transmiten en la televisión, ya que la mayor parte de las cintas que se exhiben en pantalla proceden del extranjero, principalmente de Estados Unidos. Esta situación es más que evidente, ya que mientras en el periodo de 1994 las películas mexicanas ocupaban $66 \%$ del tiempo destinado a la transmisión de este género, para el 2004 la proporción no sólo se había revertido, sino que incluso las cintas norteamericanas superaban ampliamente a las mexicanas. Durante este periodo, casi $90 \%$ del tiempo que se destinó a la presentación de películas, en las televisoras nacionales, ocurrió con base en productos de Estados Unidos.

Esta situación significa que las fórmulas gastadas durante muchos años por las empresas de televisión, donde se repetían hasta el cansancio los ciclos de películas de Pedro Infante o Cantinflas, han quedado atrás. La implementación de estrategias de venta por parte de las empresas de cine norteamericanas ha traído como consecuencia que las pantallas de la televisión nacional se inunde de películas norteamericanas, que si bien es cierto se trata de producciones más recientes que los filmes mexicanos a los que se ha hecho referencia, también es verdad que en la compra de las 
mismas se pone en marcha las políticas de venta implementadas por las grandes empresas del mundo del cine.

También conviene especificar que las películas de procedencia extranjera, a las cuales se ha hecho referencia, son presentadas casi exclusivamente en aquellos momentos en los cuales existe una baja en los índices de audiencia, ya que estas, en su mayor parte, se transmiten preferentemente durante los fines de semana o entre semana, por las noches, y en los canales de menor audiencia. Finalmente, si hacemos un ejercicio y reparamos en el tipo de contenidos a los que puede acceder el público de este país a través de la televisión abierta nacional, la respuesta sería muy simple: películas norteamericanas, magazines, telenovelas, noticieros programas de telemercadeo y uno que otro programa cómico para que los mexicanos disfruten la televisión.

\section{Bibliografía}

CASAS PÉREZ, María de la Luz (2006) Políticas públicas de comunicación en América del Norte, México: Limusa.

CROVI DRUETTA, Delia (2000) "Las industrias audiovisuales de México a partir del TLC. Una lectura desde la perspectiva del Proyecto Monarca", Razón y Palabra, núm. 19, ITESM CEM, en red: http:// www.cem.itesm.mx/dacs/publicaciones/logos/n19/19-dcrovi.html.

- (1995) "Canales abiertos de la ciudad de México: programación y preferencias del público", en Crovi Druetta (coord.) Desarrollo de las industrias audiovisuales en México y Canadá. Proyecto Monarca. México: Facultad de Ciencias Políticas y Sociales de la UNAM.

ENRÍQUEZ, Alfredo (1995) "Televisión Azteca, ¿estrategia de un esquema oculto?”, en Delia Crovi Druetta (coord.), Desarrollo de las industrias audiovisuales en México y Canadá. Proyecto Monarca, México: Facultad de Ciencias Políticas y Sociales de la UNAM.

ESTEINOU MADRID, Javier (2005) "En defensa del financiamiento de los medios públicos de comunicación”, revista electrónica Global Media Journal, vol 2, núm. 4, otoño 2005, Monterrey: ITESM.

- (2003) "La mano invisible", revista electrónica Etcétera, marzo, en red: http://www.etcetera.com.mx/.

ESTRADA, Marién (2003). "Una retrospectiva de la cinematografía en México. Historia de película...", Revista Mexicana de Comunica- 
ción, núm. 83, en red http://www.mexicanadecomunicacion.com. $\mathrm{mx} /$ Tables/RMC/rmc83/historia.html.

GARCÍA RIERA, Emilio. (1998) Breve historia del cine mexicano, primer siglo 1987-1997, IMCINE, Ed. Mapa/Canal 22/Universidad de Guadalajara.

GRANADOS CHAPA, Miguel Ángel (2002) “TV. La otra operación triunfo", columna política "Plaza Pública", Diario Reforma, primera sección, 13 de octubre, México.

INEGI, (2002). Estadísticas de cultura, INEGI, en red: http:// INEGI.com. mx. Ags. Mx.

MARTÍNEZ Garza, Francisco Javier y Lozano Rendón, José Carlos (2000) Encuestas por muestreo. Proyecto de la Cátedra Televisa. ITESM Campus Monterrey, en red: http://www.mty.itesm.mx/dhcs/ catedra/muestreo.html

MATUTE VILLASEÑOR, Pedro (2004) La perspectiva del cine mexicano en la época de la globalización, Congreso de ALAIC, Argentina: La Plata.

MEJÍA BARQUERA, Fernando (1998) "Historia mínima de la televisión mexicana (1928-1996)", en Sánchez de Armas (coord.) Apuntes para una historia de la televisión mexicana, México: Fundación Manuel Buendía.

PIEDRAS, Ernesto (2005) ¿Cuánto vale la cultura? Contribución económica de las industrias protegidas por el derecho de autor en México, México: CONACULTA, CANIEM, SOGEM, SACM.

SÁNCHEZ RUIZ, Enrique (1992) "El espacio audiovisual mexicano ante el Acuerdo de Libre Comercio Canadá-Estados Unidos-México", Comunicación y Sociedad, núm. 14-15, enero-agosto.

- (1995) "La agenda televisiva en Guadalajara", en Crovi Druetta (coord.) Desarrollo de las industrias audiovisuales en México y Canadá, Proyecto Monarca, México: Facultad de Ciencias Políticas y Sociales de la UNAM.

- (1999) "Dimensiones de la historia reciente de los medios masivos en México; algunos retos de los investigadores en comunicación para el nuevo milenio", Revista Mexicana de Comunicación, núm. 60, oct-dic., México.

- (2000a) "Industrias culturales y libre comercio. México, Canadá y 
la Unión Europea: hacia un análisis comparativo de políticas de comunicación”, revista electrónica Razón y Palabra, núm. 19, agostooctubre, en red: http://www.razonypalabra.org.mx/anterior.html.

- (2000b) "La industria audiovisual mexicana ante el TLC; radiografía de flujos desiguales", Revista Mexicana de Comunicación, núm. 61, febrero, México.

- (2003) Hollywood y su hegemonía planetaria: una aproximación histórico-estructural, La Colección de Babel, Guadalajara: Universidad de Guadalajara.

- (2004) "El audiovisual mexicano: ¿Concentrar para competir?" Global Media en español, en red: http://gmje.mty.itesm.mx

SECOFI (1993). Tratado de Libre Comercio de América del Norte, disponible en http://www.economia.gob.mx/work/snci/negociaciones/ tlcan/pdfs/tlcan $1 . p d f$

SEGOB (1999) "Ley de la Industria Cinematográfica”, Diario Oficial de la Federación, 5 de enero.

UGALDE, Víctor. (2004) "Cine mexicano uno de los perdedores con el TLC", revista electrónica Etcétera, enero, en red: http://www.etcetera.com.mx.

- (2005) "Durante 2004 hubo más recursos para el cine mexicano. Respira la pantalla grande", Revista Mexicana de Comunicación, núm. 91, febrero-marzo, en red: http://www.mexicanadecomunicacion.com.mx/Tables/RMC/rmc91/pantalla.htm1. 
